\title{
Attitudes Toward Use of Benzodiazepines among U.S. Hospice Clinicians: Survey and Review of the Literature
}

\author{
Andrew Kamell, MD ${ }^{1}$ and Lauren Kelley Smith, DO ${ }^{2}$
}

\begin{abstract}
Background: Benzodiazepines are commonly used in inpatient hospices internationally. U.S. hospice clinician views toward benzodiazepines are unknown.

Objectives: Study objectives were (1) to assess inpatient hospice clinician attitudes towards the benefits of benzodiazepines for various indications and (2) to compare these attitudes to current clinical recommendations and literature.

Methods: A survey was developed and distributed to hospices with inpatient units nationwide. Results were analyzed, then compared to current clinical guidelines. Literature review was performed. U.S. hospice physicians and nurses were the study subjects. Participants were asked to indicate their level of agreement regarding benefit from benzodiazepines for various end-of-life symptoms and to answer questions regarding benzodiazepines in delirium.

Results: Of 143 surveys returned, 128 surveys were completed. For anxiety, $80 \%$ of participants agreed that benzodiazepines were beneficial for restlessness, dyspnea $77 \%$, insomnia $68 \%$, dying process $65 \%$, agitation $57 \%$, nausea $54 \%$, hyperactive delirium $42 \%$, and severe pain $38 \%$. Nurses found benzodiazepines beneficial for more indications than physicians. Over 50\% reported benzodiazepines on their order sets for agitation, insomnia, acute anxiety, chronic anxiety, chronic panic, restlessness, seizures, and withdrawal. Among physicians, 39\% believe that benzodiazepines are overused within their own hospice. A literature review found very limited evidence of overall benefit from benzodiazepines for the symptoms listed above. In addition, this revealed significant evidence for risks and harms from benzodiazepines, particularly in patients at risk for delirium.

Conclusions: Benzodiazepines are viewed favorably by most hospice nurses and many hospice physicians for various indications, despite little supportive clinical evidence along with significant potential for harm.
\end{abstract}

\section{Introduction}

B ENZODIAZEPINES are a class of GABA agonist medications used extensively by hospice providers internationally for a variety of symptoms. ${ }^{1}$ A London inpatient hospice facility found $58 \%$ of patients were administered a benzodiazepine for indications including anxiety, agitation, and dyspnea. ${ }^{2}$ A New Zealand study of 14 hospices revealed that $75 \%$ of patients received a benzodiazepine, predominantly midazolam. ${ }^{3}$ In addition, a survey of international palliative care experts found benzodiazepines to be the most common drug prescribed by those polled to treat anxiety, agitation, and terminal restlessness in cancer patients with a life expectancy of days. ${ }^{1}$ While it would be reasonable to anticipate similar usage in the United States, the extent and frequency of benzodiazepine use in inpatient hospice settings across the United States has not been studied to date.

Unfortunately, research performed in hospice populations regarding benefits and harms of benzodiazepines is limited. Research in other populations has indicated that benzodiazepines are associated with significant harms in both short- and long-term use. ${ }^{4,5}$ As a result, best practices regarding benzodiazepines for hospice patients are unclear. ${ }^{6,7}$ Knowing this, it is critical to assess how these medications are being perceived and used by U.S. hospice providers, and to compare this to the actual evidence regarding benzodiazepine use

\footnotetext{
${ }^{1}$ OSF St. Francis Medical Center, Peoria, Illinois.

${ }^{2}$ Sangre de Cristo Hospice and Palliative Care, Pueblo, Colorado.

Accepted, January 11, 2016.
} 
in both the hospice population and in similar populations, such as the elderly and critically ill. This study aims to accomplish these goals through a survey of U.S. hospice clinicians and a review of the literature.

\section{Methods}

To assess clinician attitudes toward benzodiazepines, a survey was created using SurveyMonkey ${ }^{\circledR}$ to focus on clinician attitudes towards the appropriate use of benzodiazepines. Questions were formulated based on the literature search as well as referenced common practices by hospice providers. Subjects were asked to indicate their level of agreement on whether benzodiazepines are beneficial in treating multiple symptoms and diagnoses. Using a modified Likert scale, subjects had five levels of agreement options ranging from strongly disagree to strongly agree. Using the same scale of agreement, six questions were created asking about opinions of benzodiazepine use, specifically in treatment of delirium and delirium reversibility. Finally, symptoms and diagnoses were listed and subjects were asked to indicate if any were indications for benzodiazepines on their hospice's standard order sets. Demographic information about hospice inpatient census was requested, along with level of training and specialty of physicians participating.

Using Medicare billing criteria to select hospices for survey distribution, Hospice Analytics found 521 hospices that billed Medicare for greater than 365 days of inpatient care. Surveys were distributed to each of these hospices for which an e-mail address or fax number was available. Due to limited initial response, the survey link was also sent to all physicians on the American Academy of Hospice and Palliative Medicine (AAHPM) program director physician listserv with request to forward to inpatient hospice physicians and nurses.

The survey was available for completion from March to May 2015. Once all surveys were received, basic statistical analysis was performed. The average and range of attitudes were assessed for multiple questions. Responses were broken down to compare different response groups with total agree versus total disagree for each individual question. Using the Fisher exact test, these groups' total responses were used to calculate $p$-values. Responses were analyzed to see if there were significant differences between the following groups: clinical role (nurses versus physicians), American Board of Internal Medicine (ABIM)/American Osteopathic Board of Internal Medicine (AOBIM) certification status, hospice medical director board certified or not, fellowship trained or not, hospice inpatient unit (IPU) location if any (freestanding versus hospital based), and primary setting of practice.

A literature search was performed seeking evidence from clinical reviews and randomized controlled trials regarding benzodiazepine use in palliative and hospice patients. PubMed searches of "benzodiazepines" with either "hospice" or "palliative" together with various symptoms were used, and pertinent references within relevant articles were also evaluated. Review of the Cochrane database for "benzodiazepines" and various symptoms for evidence-based reviews as well as the National Guidelines Clearinghouse for relevant guidelines were performed and reviewed. Finally, because of the paucity of studies in hospice or palliative care, a more thorough literature review was performed for "benzodiazepines" with either "elderly," "geriatric," or "in- tensive care" (to represent populations similar to hospice) together with various symptoms.

\section{Results}

Of the 632 surveys distributed, 143 responses were returned, with 128 completing all portions. Ninety-three respondents $(73 \%)$ were physicians, of which $58(62 \%)$ were hospice medical directors, $12(13 \%)$ were associate medical directors, and $23(25 \%)$ were rounding physicians. Thirtyfour respondents $(27 \%)$ were nurses, of which 28 (82\%) were nursing leadership and $6(18 \%)$ were inpatient staff nurses. Eighty percent of physician participants were hospice and palliative medicine board certified through the ABIM/AOBIM. Only $15 \%$ of physicians that responded were certified through the hospice medical director certification board. Twenty-four percent of responding physicians had completed a fellowship in hospice and palliative medicine.

Of the 86 physician respondents to this section, 85 reported working an average 22 hours/week doing hospice, and 60 additionally worked an average of 19.8 hours/week doing palliative medicine. Sixty-six (78\%) worked an average of 15 hours/week in a hospice inpatient unit and 68 (80\%) worked an average of 14 hours/week in home hospice. Twenty-two (26\%) worked an average of 24 hours/ week in other specialties including geriatrics, hospital medicine, oncology, research, pain medicine, critical care medicine, and psychiatry. Of the hospice work, 66 (78\%) worked an average of 15 hours/week in a hospice inpatient unit and $68(80 \%)$ worked an average of 14 hours/week in home hospice.

The total participants who practice in a hospice with an inpatient facility were 104 , of whom 90 participants report performing regular inpatient hospice visits. Of the inpatient facilities, 53\% were freestanding, 35\% of inpatient facilities were hospital based, $6 \%$ were nursing home based, and $6 \%$ were based elsewhere. There was a wide range of number of general inpatient beds ranging from 1 to 76 . Seventy-eight percent of IPUs reported having a physician see patients at least five times per week, with the remaining $22 \%$ ranging from "as needed" to "every other day."

The response summaries for each question are listed in Figures 1 and 2 and Table 1. Of note, majorities of respondents agreed that benzodiazepines were beneficial for chronic anxiety and chronic panic disorder, dyspnea, insomnia, nausea and/or vomiting, agitation, terminal delirium, and the dying process. Majorities disagreed that benzodiazepines provide benefit when used for hypoactive delirium and sundowning in dementia. The responses were more evenly split for hyperactive delirium, severe pain, and acute paranoia. Regarding order sets, the majority of respondents reported that benzodiazepines were on their order set for acute anxiety (96\%), chronic anxiety, agitation, insomnia, panic, restlessness, seizure, and withdrawal, with smaller numbers reported for others.

In subgroup analysis, the most clearly and consistently significant differences were between physicians' and nurses' responses (see Table 2). Nurses' attitudes towards benzodiazepines were more for benzodiazepines utility to treat all listed symptoms except hypoactive delirium. Physicians polled had a more mixed cross-section of opinions for finding benzodiazepines useful. Thirty-nine percent felt benzodiazepines were overutilized in their own hospice. 


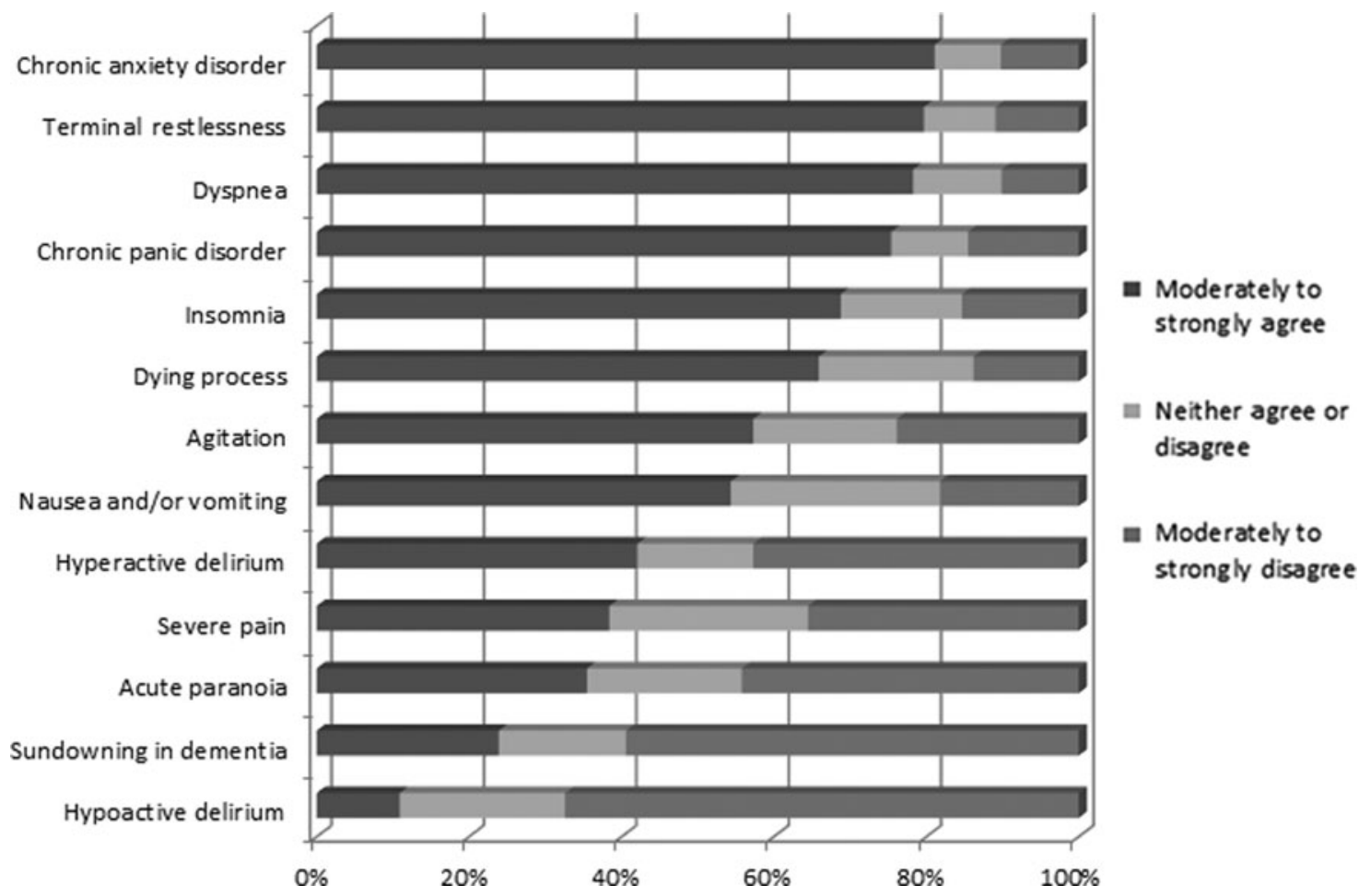

FIG. 1. Perceived Benefit of Benzodiazepines for Various Indications.

ABIM/AOBIM board certification status also appeared to be significantly related to certain responses (see Table 3 ). Also, respondents working in a freestanding hospice agreed more than hospital-based respondents that benzodiazepines are useful in treating dyspnea ( $p=0.0095)$, but were less likely to agree that benzodiazepines are one of the most important medications $(p=0.02)$. Other analyzed categories did not reach statistical significance, likely because of low numbers.

Of those who agreed that benzodiazepines are beneficial for agitation, 36\% agreed that the combination with an antipsychotic would provide added benefit and $26 \%$ disagreed.
Of those who agreed with benzodiazepines in hyperactive delirium, $45 \%$ agreed that the combination with an antipsychotic provides added benefits, while $16 \%$ disagreed. Finally, $94 \%$ of respondents chose lorazepam as their benzodiazepine of choice. Three percent of respondents chose clonazepam, $2 \%$ chose alprazolam, and $1 \%$ chose midazolam.

\section{Literature review results}

Medication appropriateness is determined based on both benefits and harms. This review aims to summarize the

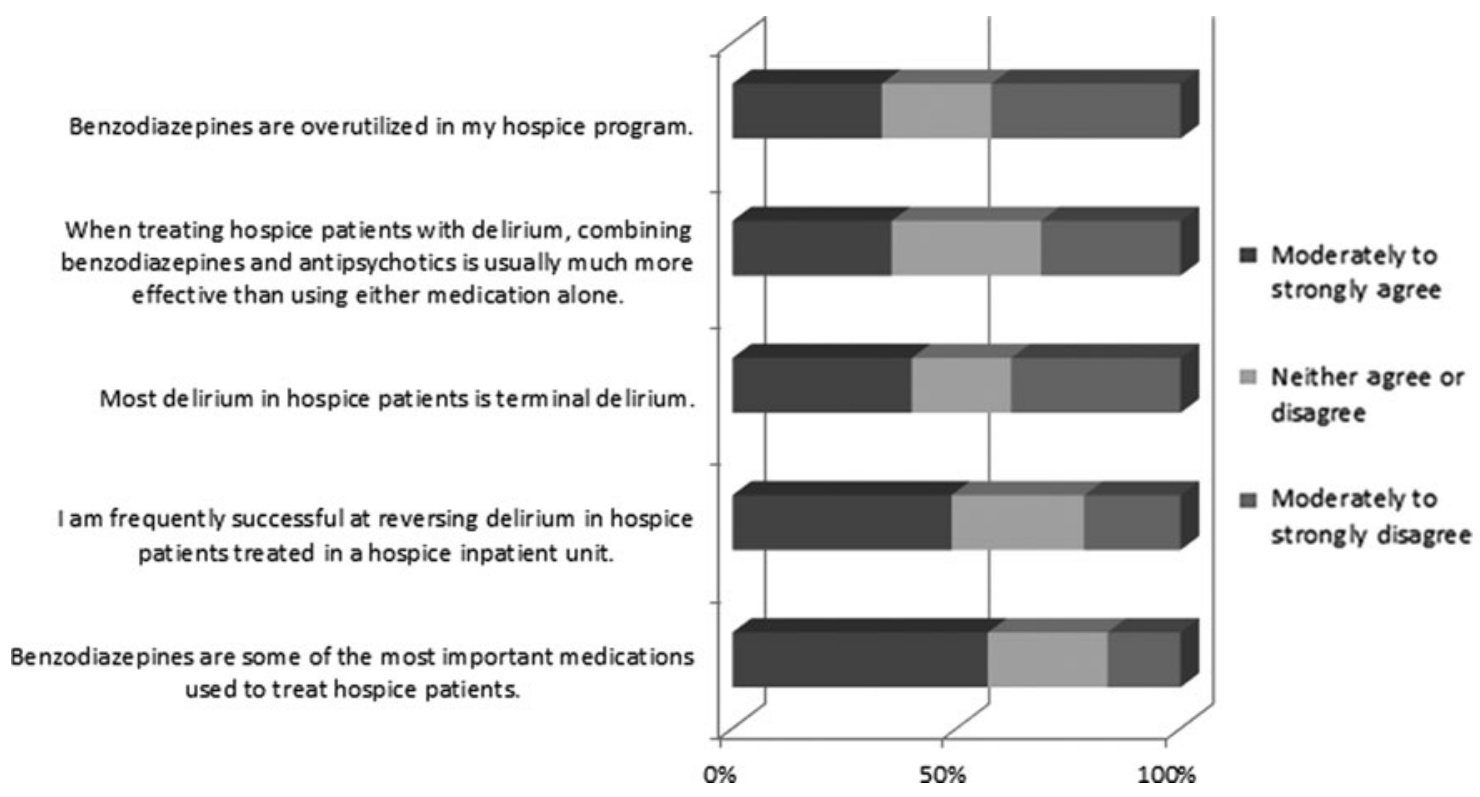

FIG. 2. Benzodiazepine and Delirium Questions. 
Table 1. Order Set Indications for BENZODIAZEPINES $(N=128)$

Benzodiazepine on my order set for

\begin{tabular}{lrr}
\hline Agitation & 80 & $65.60 \%$ \\
Acute anxiety & 117 & $95.90 \%$ \\
Chronic anxiety & 66 & $54.10 \%$ \\
Delirium & 24 & $19.70 \%$ \\
Dyspnea & 60 & $49.20 \%$ \\
Insomnia & 66 & $54.10 \%$ \\
Nausea and/or vomiting & 42 & $34.40 \%$ \\
Panic & 63 & $51.60 \%$ \\
Severe pain & 13 & $10.70 \%$ \\
Restlessness & 75 & $61.50 \%$ \\
Dying process & 35 & $28.70 \%$ \\
Seizure & 99 & $81.10 \%$ \\
Alcohol/benzodiazepine withdrawal & 69 & $56.60 \%$ \\
\hline
\end{tabular}

evidence of benefit for each symptom, as well as major risks and harms, with a focus on palliative, geriatric, and critically ill populations.

Elderly populations. Unfortunately, while evidence in the hospice population is lacking, studies in other elderly populations have demonstrated significant risks and harms from benzodiazepines. Benzodiazepines are associated with increased risk of falls, accidents, and hip fractures in the elderly. ${ }^{8}$ Benzodiazepines are associated with substantial dose-dependent worsening of physical function in the elderly, equivalent to adding three comorbid illnesses for every equivalent of benzodiazepine used..$^{9.10}$ Benzodiazepines are also associated with a dose-dependent worsening of cognitive function and memory in the elderly. ${ }^{11}$

Beers criteria gives strong recommendations based on high-quality evidence that all types of benzodiazepines should be avoided for treatment of agitation, delirium, and insomnia in geriatric patients. ${ }^{5}$ The American Medical Directors Association guidelines for delirium in the long-term care setting agree that benzodiazepines, especially ones with short half-lives, should be avoided in long-term care patients with delirium, insomnia, and psychosis, due to oversedation, rebound effects, and ineffectiveness. ${ }^{12}$ Serious concerns have been expressed about use of benzodiazepines in seriously ill older veterans. ${ }^{4}$ Therefore, it would be reasonable to require

Table 2. Physician Compared to Nurse Responses

\begin{tabular}{lccc}
\hline Benzos beneficial for & $\begin{array}{c}\text { Physicians } \\
\text { agree }\end{array}$ & $\begin{array}{c}\text { Nurses } \\
\text { agree }\end{array}$ & p value \\
\hline Chronic anxiety disorder & $80.6 \%$ & $88.2 \%$ & 0.72 \\
Chronic panic disorder & $78.5 \%$ & $73.5 \%$ & 0.76 \\
Terminal restlessness & $75.3 \%$ & $94.1 \%$ & 0.002 \\
Dyspnea & $74.2 \%$ & $82.4 \%$ & $<0.001$ \\
Insomnia & $64.5 \%$ & $82.4 \%$ & $<0.001$ \\
Dying process & $58.1 \%$ & $88.2 \%$ & 0.019 \\
Nausea and vomiting & $53.8 \%$ & $55.9 \%$ & 0.022 \\
Agitation & $46.2 \%$ & $91.2 \%$ & 1 \\
Hyperactive delirium & $35.5 \%$ & $64.7 \%$ & 0.37 \\
Severe pain & $35.5 \%$ & $50.0 \%$ & $<0.001$ \\
Acute paranoia & $33.3 \%$ & $44.1 \%$ & 0.013 \\
Sundowning in dementia & $11.8 \%$ & $61.8 \%$ & 0.018 \\
Hypoactive delirium & $6.5 \%$ & $23.5 \%$ & 0.084 \\
\hline
\end{tabular}

strong evidence of benefit before recommending their use in elderly patients.

Anxiety, panic disorders. Reviewing the literature on medications for anxiety and panic, a Cochrane review found no available studies in palliative care settings. ${ }^{6}$ The British Association for Psychopharmacology found some benzodiazepines effective for anxiety and panic treatment in the short term but with increased risk of adverse effects with any duration of treatment. ${ }^{13}$ The American Society of Clinical Oncology recommended reserving use of benzodiazepines for anxiety in cancer for a short duration if necessary, because of side effects and risks. ${ }^{14}$ The National Institute for Clinical Excellence performed a thorough literature review, and recommends against the use of benzodiazepines to treat anxiety or panic for anyone except as a short-term measure in a crisis situation. $^{15}$

Insomnia. Benzodiazepines provide some benefit in decreasing sleep latency and early arousal but suppress sleep stages 3 and 4 and REM sleep and cause adverse cognitive and psychomotor events and daytime fatigue. ${ }^{16}$ Nonbenzodiazepine sleep aids are similarly effective but generally cause less disruption to sleep architecture, and may cause less psychomotor and memory impairment, rebound insomnia, and lower risk of respiratory depression. ${ }^{17}$

A Cochrane Review found no sufficient trials that permit any firm conclusions about the use of benzodiazepines in treatment of insomnia in palliative patients. ${ }^{7}$ National Guideline Clearinghouse reviewed the evidence for benzodiazepines to treat insomnia in adults and determined that they were not recommended. ${ }^{18}$ Given the high-side effect profile of benzodiazepines and the availability of bettertolerated medication as well as nonpharmacological approaches, multiple authors suggest avoiding benzodiazepines for insomnia in older patients. ${ }^{16,19-22}$

Dyspnea. A Cochrane review of seven articles found no evidence to support benzodiazepine use to treat dyspnea, but did indicate that consideration be given to benzodiazepines as a secondary or tertiary treatment option. ${ }^{23}$ One study found an increased mortality risk for use of benzodiazepines to treat dyspnea in patients with severe respiratory disease. $^{24}$ Viola et al. compiled a review of treatments for dyspnea in cancer patients and did not recommend benzodiazepines as beneficial. ${ }^{25}$

Nausea and vomiting. Benzodiazepines are beneficial as a second-line agent for anticipatory nausea and vomiting, if behavioral therapies and aggressive initial treatment of nausea and vomiting are ineffective. ${ }^{26,27}$ For other causes of nausea, benzodiazepines appear to have minimal antiemetic effect. ${ }^{28}$ American Society of Clinical Oncology (ASCO) guidelines, based on expert opinion, suggest they could be considered only as adjunct treatment for nausea. ${ }^{29}$ However, because there are no prospective trials demonstrating benefits outside of anticipatory nausea, other authors recommend avoiding benzodiazepines entirely for nausea in the elderly population. ${ }^{28,29}$

Delirium. Hospice patients are at high risk of developing delirium for many reasons including severe illness, multiple medications, opioid use, age, dehydration. This can be very 
Table 3. Responses by ABIM/AObiM Board Certification Status

\begin{tabular}{lccccc}
\hline $\begin{array}{l}\text { Benzodiazepines } \\
\text { beneficial for }\end{array}$ & $\begin{array}{c}\text { Board certified } \\
\text { agree }\end{array}$ & $\begin{array}{c}\text { Board certified } \\
\text { disagree }\end{array}$ & $\begin{array}{c}\text { Not board } \\
\text { certified agree }\end{array}$ & $\begin{array}{c}\text { Not board } \\
\text { certified disagree }\end{array}$ & p value \\
\hline Hypoactive delirium & $4(5 \%)$ & $65(83 \%)$ & $2(17 \%)$ & $5(42 \%)$ & 0.092 \\
Sundowning in dementia & $7(9 \%)$ & $59(76 \%)$ & $4(33 \%)$ & $8(67 \%)$ & 0.06 \\
Dyspnea & $60(77 \%)$ & $9(12 \%)$ & $7(58 \%)$ & $4(33 \%)$ & 0.07 \\
Paranoia & $23(29 \%)$ & $44(56 \%)$ & $7(58 \%)$ & $3(25 \%)$ & 0.041 \\
\hline
\end{tabular}

ABIM, American Board of Internal Medicine; AOBIM, American Osteopathic Board of Internal Medicine.

distressing for both patients and families. ${ }^{30}$ Therefore, prevention and optimal treatment of delirium, as well as of potentially related symptoms such as agitation, are particularly critical.

The cornerstone palliative randomized controlled trial on treatment of delirium in hospice studied 30 terminal AIDS patients comparing haloperidol, chlorpromazine, and lorazepam. ${ }^{31}$ The study demonstrated that the patients receiving lorazepam for delirium experienced treatment-limiting side effects (including oversedation and increased confusion) which resulted in early termination of the lorazepam arm.

Multiple studies involving ICU populations, an analogous population to the inpatient hospice population, suggest that benzodiazepines increase the risk of developing and sustaining delirium as well as increase length of hospitalization and prolonged ventilation. ${ }^{32-37}$ Because benzodiazepines have been linked to the development and continuation of delirium in various populations, various authors suggest that "usual good clinical practice" is to avoid, reduce, or discontinue benzodiazepines in patients at risk for or with delirium, unless associated with substance withdrawal, seizures, palliative sedation, or extreme acute agitation. ${ }^{30,38-40}$

\section{Conclusions}

In the recent past, benzodiazepines were heavily used for sedation in the ICU setting and were used commonly within the geriatric population. However, following multiple studies involving ICU patients, other medications and strategies have largely replaced benzodiazepines in the intensive care setting. ${ }^{37}$ At the same time, various studies have demonstrated significant harms in the elderly and those at risk for delirium. Guidelines increasingly recommend avoiding use in those populations as well. 5,30,38,39 Until more hospice-oriented evidence is available, strong regard should be paid to studies within the intensive care and geriatric populations, which are similar in many ways to the inpatient and home hospice populations, respectively.

This study demonstrates that large percentages of hospice clinicians view benzodiazepines as beneficial for a wide range of symptoms. Specifically, majorities of respondents agreed that benzodiazepines were beneficial for chronic anxiety and chronic panic disorder, dyspnea, insomnia, nausea and/or vomiting, agitation, terminal restlessness, and the dying process, with pluralities reporting benefits for hyperactive delirium, severe pain, and acute paranoia. In addition, the majority of respondents reported that benzodiazepines were on their order sets for acute anxiety, chronic anxiety, agitation, insomnia, panic, restlessness, seizure, and withdrawal, with substantial minorities for most other problems. It is reasonable to infer that use of benzodiazepines for these indications is common practice among hospice providers in the United States.

Our review of the literature provides support for benefits from benzodiazepines in alcohol withdrawal, initial seizure treatment, anticipatory nausea, and in crisis situations for acute anxiety. Our literature review failed to find evidence to support overall benefit for most of the symptoms listed, and found evidence of risks and harms from benzodiazepine use, particularly in elderly patients, those at risk for delirium, and in longer-term use. ${ }^{5}$

A special focus of this study was on benzodiazepines in delirium. Given that delirium is frequently underdiagnosed, ${ }^{40-42}$ the authors considered some symptoms, specifically restlessness, agitation, acute paranoia, and the dying process, to be potential proxy responses for patients who may have delirium. Overall, large percentages of hospice clinicians in this survey reported benzodiazepines as beneficial in many patients who may have delirium. Given the cornerstone study within the palliative field, ${ }^{31}$ available literature, ${ }^{30}$ and the evidence suggesting that benzodiazepines may cause or worsen delirium, ${ }^{33,39,43}$ the numbers of positive responses are striking. In addition, considering that antipsychotics are considered first-line treatments, ${ }^{45}$ it is also remarkable that only $45 \%$ of those reporting benefit from benzodiazepines in hyperactive delirium indicated that combination with an antipsychotic would provide added benefit in treatment of delirium.

It was also notable that consistently more nurses than physicians reported that benzodiazepines are beneficial for various symptoms. This is especially relevant, since hospice nurses may have freedom to implement orders from order sets without consulting a physician. Of note, $39 \%$ of physicians and $8 \%$ of nurses agreed that benzodiazepines are overused within their own hospice. It is possible that the difference in attitudes between nurses and physicians may contribute to higher rates of benzodiazepine use than hospice physicians would prefer.

Weaknesses of this study include the limited response rates leading to a relatively small sample size, with most questions having an estimated margin of error of $9 \%$. The respondents are not a completely random sample, partly because certain groups might have been more likely to respond, and partly because using the AAHPM listserv may have skewed distribution towards providers more connected to AAHPM. Also, this study surveyed attitudes and beliefs, which may not precisely reflect prescribing patterns or the actual contents of order sets. The focus on U.S. hospices with inpatient units may also limit the generalizability of this study. In addition, the literature review, while extensive, was not comprehensive.

This study shows that many hospice physicians and most hospice nurses view benzodiazepines as beneficial treatments 
for a wide variety of symptoms including patients with delirium. However, the accompanying review largely failed to find literature that supports these views, and instead found evidence that benzodiazepines should be avoided in most patients at risk for delirium. This apparent disconnect between attitudes toward benzodiazepines and available research in similar populations suggests that these attitudes rely primarily upon anecdotal experience rather than clinical evidence of efficacy. ${ }^{46}$ The fact that hospice patients generally have a limited life expectancy and a high risk of delirium requires us to be particularly cognizant of potential harms from medications and to ensure the best-quality evidencebased care. This study demonstrates an urgent need for highquality hospice and palliative-based research to evaluate the benefits and harms of benzodiazepines in this population, and to determine appropriate indications for their use. It also reveals the need to ensure individual hospice physicians and nurses are aware of and following current best practices. In the meantime, it would be reasonable to utilize restraint with benzodiazepine prescribing, particularly in patients at risk for delirium, and to consider adoption of strategies used in the geriatric and intensive care settings to minimize the need for these medications.

\section{Acknowledgments}

Thank you to Parkview Medical Center, Pueblo, Colorado, for support. Thank you to Hospice Analytics for support and assistance with distribution of the survey. Thank you to Palliative Department of University of Colorado at Denver for of feedback and suggestions on the research proposal.

\section{Author Disclosure Statement}

No competing financial interests exist for either author.

\section{References}

1. Lindqvist O, Lundquist G, Dickman A, et al.: Four essential drugs needed for quality care of the dying: A Delphi-study based international expert consensus opinion. J Palliat Med 2013;16:38-43.

2. Henderson M, MacGregor E, Sykes N, Hotopf M: The use of benzodiazepines in palliative care. Palliat Med 2006;20: 407-412.

3. Ensor B, Cohen D: Benchmarking benzodiazepines and antipsychotics in the last 24 hours of life. N Z Med J 2012; 125:19-30.

4. Garrido MM, Prigerson HG, Penrod JD, et al.: Benzodiazepine and sedative-hypnotic use among older seriously Ill veterans: Choosing wisely? Clin Ther 2014;36:1547-1554.

5. American Geriatrics Society 2012 Beers Criteria Update Expert Panel: American Geriatrics Society updated Beers Criteria for potentially inappropriate medication use in older adults. J Am Geriatr Soc 2012;60:616-631.

6. Candy B, Jackson KC, Jones L, et al.: Drug therapy for symptoms associated with anxiety in adult palliative care patients. Cochrane Database Syst Rev 2012;10:CD004596.

7. Hirst A, Sloan R: Benzodiazepines and related drugs for insomnia in palliative care. Cochrane Database Syst Rev 2002:CD003346.

8. Peron EP, Gray SL, Hanlon JT: Medication use and functional status decline in older adults: A narrative review. Am J Geriatr Pharmacother 2011;9:378-391.
9. Hilmer SN, Mager DE, Simonsick EM, et al.: A drug burden index to define the functional burden of medications in older people. Arch Intern Med 2007;167:781-787.

10. Carrière I, Mura T, Pérès K, et al.: Elderly benzodiazepine users at increased risk of activity limitations: Influence of chronicity, indications, and duration of action: The threecity cohort. Am J Geriatr Psychiatry 2014. (E-pub ahead of print.)

11. Tannenbaum C, Paquette A, Hilmer S, et al.: A systematic review of amnestic and non-amnestic mild cognitive impairment induced by anticholinergic, antihistamine, GABAergic and opioid drugs. Drugs Aging 2012;29:639-658.

12. AMDA: Delirium and Acute Problematic Behavior in the Long-term Care Setting. American Medical Directiors Association, 2008.

13. Baldwin DS, Anderson IM, Nutt DJ, et al:: Evidencebased pharmacological treatment of anxiety disorders, posttraumatic stress disorder and obsessive-compulsive disorder: A revision of the 2005 guidelines from the British Association for Psychopharmacology. J Psychopharmacol 2014;28:403-439.

14. Andersen BL, DeRubeis RJ, Berman BS, et al.: Screening, assessment, and care of anxiety and depressive symptoms in adults with cancer: An American Society of Clinical Oncology guideline adaptation. J Clin Oncol 2014;32:1605-1619.

15. For Mental Health NCC, for Primary Care NCC, Others. Generalised Anxiety Disorder and Panic Disorder (with or without Agoraphobia) in Adults: Management in Primary, Secondary and Community Care. National Institute for Health and Clinical Excellence, 2011.

16. Wolkove N, Elkholy O, Baltzan M, Palayew M: Sleep and aging: 2. Management of sleep disorders in older people. CMAJ 2007;176:1449-1454.

17. Wagner J, Wagner ML: Non-benzodiazepines for the treatment of insomnia. Sleep Med Rev 2000;4:551-581.

18. National Guideline Clearinghouse: Clinical Guideline for the Treatment of Primary Insomnia in Middle-aged and Older Adults. www.guideline.gov/content.aspx $? \mathrm{id}=48218 \&$ search $=$ clinical+guideline+for+the+treatment+of+primary+insomnia+ in+middle-aged. (Last accessed June 19, 2015.)

19. Bloom HG, Ahmed I, Alessi CA, et al.: Evidence-based recommendations for the assessment and management of sleep disorders in older persons. J Am Geriatr Soc 2009; 57:761-789.

20. Kyme C: Risks could outweigh the benefits of sedative hypnotics for insomnia in the elderly. Nat Rev Neurol 2006;2:123-123.

21. Brandt NJ, Piechocki JM: Treatment of insomnia in older adults: Re-evaluating the benefits and risks of sedative hypnotic agents. J Gerontol Nurs 2013;39:48-54.

22. Glass J, Lanctôt KL, Herrmann N, et al.: Sedative hypnotics in older people with insomnia: Meta-analysis of risks and benefits. BMJ 2005;331:1169.

23. Simon ST, Higginson IJ, Booth S, et al.: Benzodiazepines for the relief of breathlessness in advanced malignant and non-malignant diseases in adults. Cochrane Database Syst Rev 2010:CD007354.

24. Ekström MP, Bornefalk-Hermansson A, Abernethy AP, Currow DC: Safety of benzodiazepines and opioids in very severe respiratory disease: National prospective study. BMJ 2014;348:g445.

25. Viola R, Kiteley C, Lloyd NS, et al.: The management of dyspnea in cancer patients: A systematic review. Support Care Cancer 2008;16:329-337. 
26. Kamen C, Tejani MA, Chandwani K, et al.: Anticipatory nausea and vomiting due to chemotherapy. Eur J Pharmacol 2014;722:172-179.

27. Razavi D, Delvaux N, Farvacques C, et al.: Prevention of adjustment disorders and anticipatory nausea secondary to adjuvant chemotherapy: A double-blind, placebo-controlled study assessing the usefulness of alprazolam. J Clin Oncol 1993;11:1384-1390.

28. Glare P, Miller J, Nikolova T, Tickoo R: Treating nausea and vomiting in palliative care: A review. Clin Interv Aging 2011; 6:243-259.

29. Basch E, Prestrud AA, Hesketh PJ, et al.: Antiemetics: American Society of Clinical Oncology clinical practice guideline update. J Clin Oncol 2011;29:4189-4198.

30. Bush SH, Leonard MM, Agar M, et al.: End-of-life delirium: Issues regarding recognition, optimal management, and the role of sedation in the dying phase. J Pain Symptom Manage 2014;48:215-230.

31. Breitbart W, Marotta R, Platt MM, et al.: A double-blind trial of haloperidol, chlorpromazine, and lorazepam in the treatment of delirium in hospitalized AIDS patients. Am J Psychiatry 1996;153:231-237.

32. Pandharipande P, Shintani A, Peterson J, et al.: Lorazepam is an independent risk factor for transitioning to delirium in intensive care unit patients. Anesthesiology 2006;104: 21-26.

33. Pandharipande P, Cotton BA, Shintani A, et al.: Prevalence and risk factors for development of delirium in surgical and trauma intensive care unit patients. J Trauma 2008;65: 34-41.

34. Agarwal V, O’Neill PJ, Cotton BA, et al.: Prevalence and risk factors for development of delirium in burn intensive care unit patients. J Burn Care Res 2010;31:706-715.

35. Pandharipande PP, Pun BT, Herr DL, et al.: Effect of sedation with dexmedetomidine vs lorazepam on acute brain dysfunction in mechanically ventilated patients: The MENDS randomized controlled trial. JAMA 2007;298: 2644-2653.

36. Riker RR, Shehabi Y, Bokesch PM, et al. Dexmedetomidine vs midazolam for sedation of critically ill patients: A randomized trial. JAMA 2009;301:489-499.
37. Barr J, Fraser GL, Puntillo K, et al.: Clinical practice guidelines for the management of pain, agitation, and delirium in adult patients in the intensive care unit. Crit Care Med 2013;41:263-306.

38. Clegg A, Young JB: Which medications to avoid in people at risk of delirium: A systematic review. Age Ageing 2011;40:23-29.

39. Meagher D, Adamis D, Trzepacz P, Leonard M: Features of subsyndromal and persistent delirium. $\mathrm{Br} \mathrm{J}$ Psychiatry 2012;200:37-44.

40. Bush SH, Bruera E: The assessment and management of delirium in cancer patients. Oncologist 2009;14:10391049.

41. Moyer DD: Terminal delirium in geriatric patients with cancer at end of life. Am J Hosp Palliat Med 2011;28: $44-51$.

42. Rice KL, Bennett M, Gomez M, et al.: Nurses' recognition of delirium in the hospitalized older adult. Clin Nurse Spec 2011;25:299-311.

43. Jackson N, Doherty J, Coulter S. Neuropsychiatric complications of commonly used palliative care drugs. Postgrad Med J 2008;84:121-126; quiz 125.

44. Gaudreau J-D, Gagnon P, Harel F, et al.: Psychoactive medications and risk of delirium in hospitalized cancer patients. J Clin Oncol 2005;23:6712-6718.

45. Ross DD, Alexander CS: Management of common symptoms in terminally ill patients: Part II. Constipation, delirium and dyspnea. Am Fam Physician 2001;64:1019-1027.

46. Croskerry P: The importance of cognitive errors in diagnosis and strategies to minimize them. Acad Med 2003;78: $775-780$.

Address correspondence to:

Lauren Kelley Smith, DO

Sangre de Cristo Hospice and Palliative Care

1207 Pueblo Boulevard Way

Pueblo, CO 81005

E-mail: lalabighair@gmail.com 